\title{
Vascular ventricular coupling in patients with malignant phase hypertension: the West Birmingham malignant hypertension project
}

\author{
Alena Shantsila ${ }^{1}$, Girish Dwivedi ${ }^{1}$, Eduard Shantsila ${ }^{1}$, Richard P Steeds ${ }^{2}$, Gareth Beevers ${ }^{1}$ and \\ Gregory YH Lip ${ }^{1}$
}

Patients with malignant hypertension (MHT) have persistent vascular dysfunction and a much worse clinical prognosis than non-MHT hypertensive patients, despite good long-term blood pressure (BP) control. We hypothesized that abnormal arterial (arterial elastance (Ea); arterial elastance index (Eal)) and ventricular (End-systolic elastance (Ees) and End-diastolic elastance (Eed)) elastances are present in treated MHT patients, compared with non-MHT hypertensive controls. Echocardiographic parameters of cardiac and vascular stiffness (Eal, Ees and Eed) were quantified in patients with stable MHT and treated 'high-risk' hypertension patients (HHT, but non-MHT). All patients had well-controlled BP, with a median follow-up time for MHT of 144 months. Ea was calculated from stroke volume and systolic BP and adjusted by body area (Eal). Ees was calculated using systolic and diastolic BP, stroke volume, ejection fraction, time intervals and estimated normalized ventricular elastance at arterial end diastole. Eed was calculated from Doppler parameters and the diastolic filling volume. Both study groups had preserved left ventricular contractility, with no significant differences on 3D-echocardiography $(P=0.10)$ There were no significant differences in Eal $(P=0.83)$, Ees $(P=0.32)$, Eed $(P=0.23)$ and arterial-ventricular interaction (Ees/Ea, $P=0.69)$. In the MHT group, Eed positively correlated with age $(r=0.56, P=0.38)$ and systolic $\mathrm{BP}(r=0.68, P=0.008)$. On multivariable regression analysis, MHT status was not predictive of the ventricular and Ea. Despite documented vascular dysfunction in patients with previously diagnosed stable MHT, the arterial and systolic elastances were similar to HHT patients, suggesting that adequate BP control in MHT patients allows preservation or restoration of normal arterial-ventricular coupling.

Hypertension Research (2012) 35, 725-728; doi:10.1038/hr.2012.18; published online 23 February 2012

Keywords: arterial elastance; high risk hypertension; malignant phase hypertension; ventricular-arterial couplings; ventricular elastance

\section{INTRODUCTION}

Impaired elastic properties of the heart and vessels are emerging as critical elements of cardiovascular pathology. It is accepted that large arteries are not merely passive conduits of blood but rather contribute actively to a variety of cardiovascular functions. ${ }^{1}$ The stiffening of large arteries parallels arterial hypertension and is linked to accelerated atherosclerosis. ${ }^{1}$ Similarly, abnormalities of cardiac relaxation alter intracardiac hemodynamics and are a key pathophysiological component of heart failure with preserved systolic function. ${ }^{2-3}$

Arterial and cardiac stiffness are determined by various factors, such as the composition of the extracellular matrix, the tonus of arterial smooth muscles and the relaxation pattern of cardiomyocytes. Progressive hyperplasia/hypertrophy of smooth and cardiac muscle and excessive collagen deposition are directly involved in a self-perpetuated vicious cycle of high-stiffness-associated cardiovascular dysfunction (for example, hypertension and cardiac diastolic dysfunction). ${ }^{4}$ Large arteries with preserved elastic properties are able to stretch during systole, thus retaining some of the blood volume to be returned to the circulation during diastole. This reduces cardiac cycle-dependent fluctuations in blood pressure (BP), reduces cardiac afterload and improves coronary perfusion pressure during diastole. Increased vascular and left ventricular (LV) stiffening on the other hand may lead to an alteration in ventricular-arterial coupling, which can be assessed reproducibly by echocardiographic measurement of arterial and cardiac elastance. ${ }^{5,6}$ Indeed, in patients with the same systolic function, an adverse prognosis can be identified by susceptibility to a loading change, such as an increased preload or afterload. ${ }^{5}$ This vulnerability to an acute loading change is determined by ventricular stiffness and the end-systolic ventriculo-arterial interaction. ${ }^{6}$

Malignant phase hypertension (MHT) is the most severe form of hypertension associated with poor prognosis despite modern antihypertensive treatment. ${ }^{7}$ We have recently shown that MHT patients

1University of Birmingham Centre for Cardiovascular Sciences, City Hospital, Birmingham, UK and 2Department of Cardiology, University Hospital Birmingham NHS Foundation Trust, Birmingham, UK

Correspondence: Professor GYH Lip, University of Birmingham, Centre for Cardiovascular Sciences, City Hospital, Birmingham B18 7QH, UK.

E-mail: g.y.h.lip@bham.ac.uk

Received 2 November 2011; accepted 29 December 2011; published online 23 February 2012 
have a number of specific pathophysiological features, which differ from those of non-MHT patients, such as persistent endothelial dysfunction and increased pulse wave velocity despite satisfactory BP control. ${ }^{8}$ Although the abnormalities of arterial and cardiac properties in essential hypertension are well recognized, the status of arterial and cardiac elastance in treated MHT have not yet been explored.

In this study, we tested the hypothesis that abnormal arterial (arterial elastance (Ea); arterial elastance index (EaI)) and ventricular (end-systolic elastance (Ees) and end-diastolic elastance (Eed)) elastances are present in treated MHT patients, compared with non-MHT hypertensive controls.

\section{METHODS}

We recruited consecutive eligible patients with a prior history of confirmed MHT. The diagnosis of MHT was clinically defined by severe hypertension with the characteristic ocular fundal changes detected by fundoscopy and retinal photography: bilateral retinal hemorrhages, cotton wool spots or exudates, with or without papilloedema. ${ }^{9}$ Peak levels of BP in the MHT group have been registered at presentation and were $238 \pm 20.8 \mathrm{~mm} \mathrm{Hg}$ for systolic BP and $157 \pm 19.2 \mathrm{~mm} \mathrm{Hg}$ for diastolic BP, but the BP was generally well controlled with mean systolic and diastolic BP during follow-up since the diagnosis $138.1 \pm 10.1 \mathrm{~mm} \mathrm{Hg}$ and $88.0 \pm 6.2 \mathrm{~mm} \mathrm{Hg}$, respectively. The average duration of MHT prior the recruitment was $144 \pm 108$ months.

Initially we identified a group of 101 with MHT from our MHT database who met the study inclusion criteria. ${ }^{8}$ Of these, 40 patients undergoing regular outpatient follow-up did not have exclusion criteria specified below and were invited to take part in the study - only 15 patients consented to take part, which fulfilled our a power calculation (see below, $n=15$ needed in the MHT group). The relatively small proportion of patients consented for the study was thought to be because of planned stress myocardial contrast echocardiography to exclude coronary artery disease required as part of the study protocol. ${ }^{8}$ The study was approved by the local research ethics committee and written informed consent has been obtained from all participants.

Patients with MHT were compared with 28 age and consecutively recruited sex matched patients with treated 'high-risk' hypertension (HHT) as 'disease controls'. As in recent clinical trials, ${ }^{10}$ HHT was defined by the presence of established hypertension (but non-MHT) with one or more of the following cardiovascular risk factors: LV hypertrophy using Sokolow-Lyon or Cornell voltage criteria on 12-lead electrocardiogram, age $>55$ years, peripheral vascular disease or with a known family history of coronary artery disease. We excluded subjects with known underlying renal, renovascular or adrenal causes for their hypertension. Other exclusion criteria were coronary artery disease, valvular heart disease, LV dysfunction (ejection fraction $<50 \%$ ), diabetes mellitus, liver disease, serum creatinine $>200 \mu \mathrm{moll}^{-1}$, malignancy, recent ( $<3$ months) arterial or venous thromboembolic disease, active infections and/or a history of inflammatory or connective tissue disorders.

All study subjects abstained from smoking, alcohol, tea and coffee for $24 \mathrm{~h}$ before the study. Hypertensive subjects were advised to omit their medications on the study day, as prolonged treatment omission was deemed unethical. All scans were performed in a quiet, darkened, temperature controlled room after patient rested for $15-20 \mathrm{~min}$.

\section{Echocardiography}

All subjects underwent M-mode, two-dimensional echocardiography, tissue Doppler imaging and three-dimensional (3D) echocardiography using Phillips iE33 ultrasound machine (Bothel, WA, USA). Modern off-line QLAB software (Xcelera, Phillip (iE33) Ultrasound Quantification Module) was used for quantification of LV function. All parameters were measured in triplicate and averaged. Two-dimensional and color Doppler imaging was performed to screen for hemodynamically significant (more than moderate regurgitation or more than mild stenosis or prosthetic valve) valve disease. LV ejection fraction was assessed by 3D echocardiography. Measurements of arterialvascular interaction were made in accordance with previously validated methodologies. ${ }^{11,12}$ To calculate effective EaI we calculated effective Ea from stroke volume (by 3D echocardiography) and systolic BP and adjusted by body area. Ees was calculated using systolic and diastolic BP, stroke volume, preejection and total-systolic times. End-diastolic elastance (Eed) was estimated from the ratio of mitral inflow early-diastolic filling velocity to the mitral annular early-diastolic tissue Doppler imaging velocity (E') divided then by volume filling during diastole. In the absence of significant aortic regurgitation, stroke volume can be used as an indicator of ventricular filling volume. The ratio arterial-ventricular interaction (Ea/Ees) was calculated. The inter- and intra-observer variability for echocardiography parameters was assessed $(n=10)$ and calculated as $11 \%$ and $6.8 \%$, respectively.

\section{Power calculations}

On the basis on our previous work on arterial stiffness, preliminary analysis of parameters of elastance in normotensive individuals, we calculated that a sample size of at least 15 patients in each group would have an $80 \%$ power to detect a significant difference $\geqslant 0.5$ s.d. ${ }^{8}$

\section{Statistical analyses}

Data are expressed as mean \pm s.d. for normally distributed data; or median and inter-quartile range for descriptive and/or non-normally distributed data. Comparisons between MHT and HHT were performed by $t$-test (for normally distributed variables) or Mann-Whitney test (for non-normally distributed variables). Correlation analysis was performed using Pearson's method for normally distributed parameters and Spearman's method for non-normally distributed variables. Stepwise linear regression analyses were used for multivariate analyses. Using univariate linear regression analysis, we established individual predictors of the parameters of elastance including the predictive value of MHT vs. HHT in the total study population. This was followed by stepwise multivariate analysis, where predictive role of MHT $v s$. HHT status for the elastance parameters was assessed after the adjustment for age plus significant predictors identified by the univariate analysis. A $P$-value of $<0.05$ was considered statistically significant. SPSS 17 (SPSS Inc, Chicago, IL, USA) statistical software was used to perform the statistical analyses.

\section{RESULTS}

We recruited 15 MHT patients (mean age $53.1 \pm 12.9$ years, $73 \%$ male) and 28 HHT patients (age $47.9 \pm 12.2$ years, $61 \%$ male). The two study groups were comparable in age, sex and body mass index (Table 1). The hypertensive groups had a similar profile of cardiovascular risk factors, and systolic and diastolic BP. Both study groups had preserved LV contractility $(P=0.10)$ MHT vs. HHT on 3D echocardiography.

\section{Vascular-ventricular interactions}

No significant differences were observed between the two groups in EaI $(P=0.83)$, Ees $(P=0.32)$, Eed $(P=0.23)$ or arterial-ventricular interaction $(\mathrm{Ea} / \mathrm{Ees}, P=0.69)$ (Table 2$)$. In the MHT group, Eed was positively correlated with age $(r=0.56, P=0.38)$ and systolic BP $(r=0.68, P=0.008)$ (Figure 1).

\section{Univariate and multivariate analysis}

Univariate analyses for the predictors of elastance parameters are shown in Table 3. On multivariate stepwise linear regression analysis of the predictive value of MHT vs. HHT status for the elastance parameters, MHT status was not predictive of Ea/Ees ( $\beta$ coefficient $0.70, P=0.63$ ), EaI ( $\beta$ coefficient $0.13, P=0.30$ ), EeS ( $\beta$ coefficient $0.04, P=0.73)$ or EsD ( $\beta$ coefficient $-0.30, P=0.84$ ).

\section{DISCUSSION}

In this study we show for the first time that the cardiac and Eas in patients with controlled MHT are comparable to those with 'high-risk' hypertension. Thus, despite documented vascular dysfunction in patients with previously diagnosed stable MHT, the arterial and ventricular elastances were found to be similar to 'high-risk' nonMHT hypertensive patients. 
The method used in the study represents an advance in the assessment of elastic cardiovascular properties. This echocardiographic approach has been validated by direct comparisons with invasive analysis of pressure-volume curves. ${ }^{11,12}$ The non-invasive nature of the method makes it suitable for clinical use and for monitoring of treatment effectiveness, leading to its growing popularity among researchers.

Measures of Ea reflect the artery's ability to expand and contract in synchrony with cardiac pulsation and relaxation. Cardiac elastance depends on different factors such as increased LV mass, reduced myocyte content, increased collagen deposition and cross-linking. ${ }^{13}$ Additionally, cardiac elastance is affected by LV systolic contraction and diastolic relaxation. This is particularly relevant to the hypertensive heart, when passive structural and geometric changes significantly increase Ees. The hypertensive heart is also characterized by progressive fibrosis and cross-linking of collagen as well as by vascular stiffening. ${ }^{14,15}$ At a certain stage, these abnormalities result in increased BP, cardiac afterload and diastolic dysfunction. ${ }^{15,16}$ These changes are also reflected by elevation in the end-systolic pressurevolume ratio accompanied by a rise in Ees and a reduction in stroke volume and further progression of the diastolic dysfunction via impaired and raised diastolic pressure. ${ }^{17}$ Additionally, high Ees accompanied by diastolic dysfunction can increase myocardial oxygen consumption, thus limiting myocardial perfusion reserve. ${ }^{15}$ Of interest, the results of this study showing no significant change in Ees in MHT vs. essential hypertension, which accords with the previous observation of preserved myocardial perfusion reserve in MHT despite impairment of endothelial dysfunction. ${ }^{8}$

The Ea/Ees ratio represents ventriculo-arterial stiffening or afterload-adjusted contractility of the cardiovascular system. ${ }^{16}$ Appropriate coupling of the heart to the artery can be interpreted in several ways.

Table 1 Demographic and clinical characteristics of the study groups

\begin{tabular}{lccc}
\hline & MHT $(\mathrm{n}=15)$ & HHT $(\mathrm{n}=28)$ & P-value \\
\hline Age, years & $53.1 \pm 12.9$ & $47.9 \pm 12.2$ & 0.30 \\
Sex, $n(\%)$ & $11(73)$ & $17(61)$ & 0.41 \\
Systolic BP, mm Hg & $149.3 \pm 20.3$ & $148.4 \pm 22.8$ & 0.95 \\
Diastolic BP, $\mathrm{mm} \mathrm{Hg}^{-2}$ & $87.7 \pm 10.5$ & $84.0 \pm 9.5$ & 0.66 \\
Body mass index, $\mathrm{kg} \mathrm{m}^{-2}$ & $29.8 \pm 5.56$ & $30.1 \pm 6.37$ & 0.86 \\
Total cholesterol, $\mathrm{mmol} \mathrm{I}^{-1}$ & $4.84 \pm 0.79$ & $5.22 \pm 1.04$ & 0.23 \\
Creatinine, $\mu \mathrm{mol} \mathrm{I}^{-1}$ & $118 \pm 39.9$ & $83.3 \pm 26.1$ & 0.002 \\
Glucose, mmol I-1 & $5.25 \pm 0.53$ & $5.13 \pm 0.80$ & 0.63 \\
Smoking, $n(\%)$ & $6(40)$ & $12(43)$ & 0.86 \\
\hline
\end{tabular}

Abbreviations: BP, blood pressure; HHT, 'high-risk' hypertension; MHT, malignant hypertension. Values expressed as mean \pm s.d. or median.
Firstly, it can be considered as effective transfer of blood from the heart to the peripheral circulation without excessive changes in BP. Secondly, it can be viewed as an optimal cardiovascular flow reserve for a defined arterial pressure. The interaction of the heart with the arterial system (ventricular-arterial coupling) is a key determinant of cardiovascular performance. ${ }^{16,18} \mathrm{EaI}$ reflects both mean resistive and pulsatile load and it is influenced by the heart rate.

Ventricular-arterial coupling is expressed by the Ea/Ees ratio. ${ }^{18}$ Under normal circumstances, the Ea/Ees ratio varies from 0.5 to 1.0 , a range where cardiac work and efficiency are optimized. ${ }^{16,18}$ It is well recognized that changes in contractile performance alter Ees, but Ees is also influenced by chamber geometry and by factors that alter the passive stiffness of the myocardium. ${ }^{11,18,19}$ The values for the Ea/Ees ratio obtained in both study groups are within the above range and
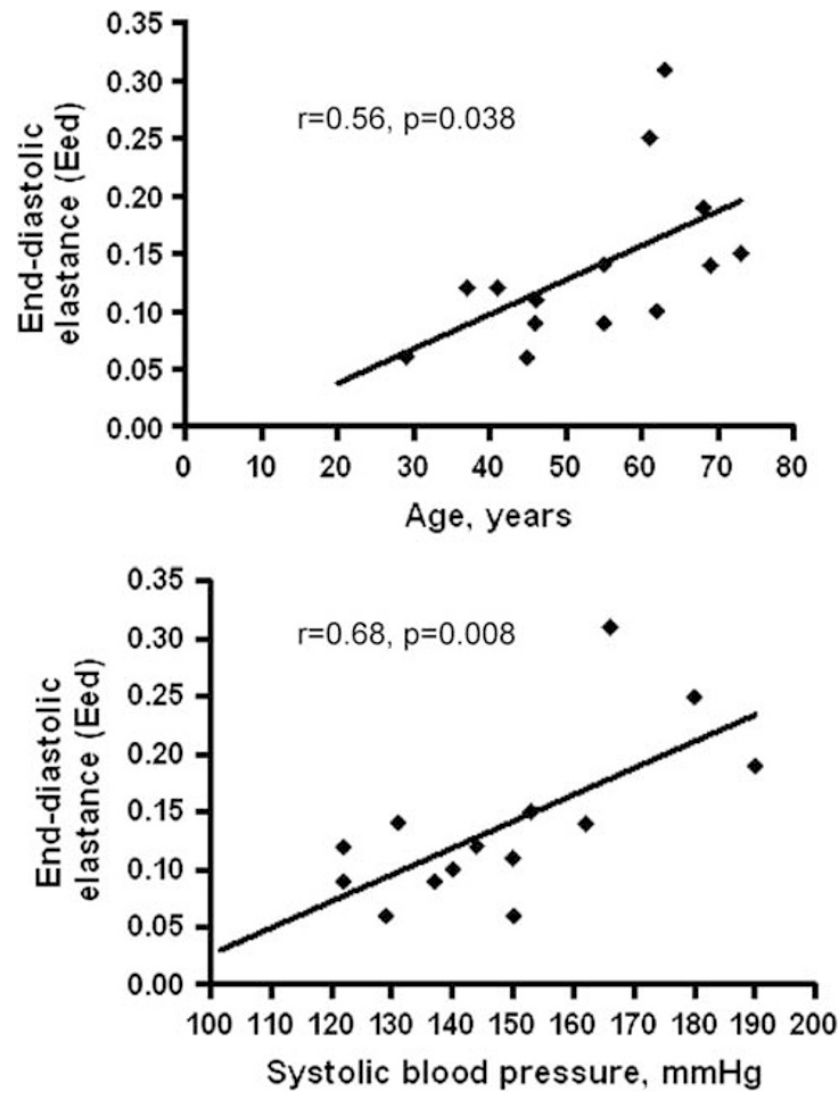

Figure 1 Correlation of Eed with age and systolic BP in MHT.

Table 2 Parameters of cardiac function and vascular and left ventricular elastance

\begin{tabular}{|c|c|c|c|}
\hline & $M H T(\mathrm{n}=15)$ & $H H T(\mathrm{n}=28)$ & P-value \\
\hline Ejection fraction 3D, \% & $63.5 \pm 6.4$ & $67.5 \pm 6.8$ & 0.098 \\
\hline Cardiac output index, I $\mathrm{min}^{-1} \mathrm{~m}^{-2}$ & $2.2 \pm 0.6$ & $1.9 \pm 0.54$ & 0.92 \\
\hline Arterial elastance index (Eal, $\mathrm{mm} \mathrm{Hg} \mathrm{ml}^{-1} \mathrm{~m}^{-2}$ ) & $1.19 \pm 0.6$ & $1.33 \pm 0.47$ & 0.83 \\
\hline End-systolic elastance (Ees, $\mathrm{mm} \mathrm{Hg} \mathrm{ml}^{-1}$ ) & $2.97 \pm 1.97$ & $3.19 \pm 1.25$ & 0.32 \\
\hline End-diastolic elastance (Eed) & $0.12(0.09-0.16)$ & $0.11(0.08-0.13)$ & 0.23 \\
\hline $\mathrm{Ea} / \mathrm{Ees}$ & $0.87 \pm 0.2$ & $0.84 \pm 0.19$ & 0.69 \\
\hline
\end{tabular}

Abbreviations: HHT, 'high-risk' hypertension; MHT, malignant hypertension.

Values expressed as mean \pm s.d. or median. 
Table 3 Univariate linear regression analysis of the predictive value of MHT vs. HHT status for the elastance parameters

\begin{tabular}{|c|c|c|c|c|c|c|c|c|}
\hline \multirow[b]{2}{*}{ Variable } & \multirow{2}{*}{$\begin{array}{c}\text { Ea/Ees } \\
\beta \text { coefficient }\end{array}$} & \multicolumn{3}{|c|}{ Eal } & \multirow{2}{*}{$\begin{array}{c}\text { EeS } \\
\beta \text { coefficient }\end{array}$} & \multicolumn{3}{|c|}{$E e D\left(\mathrm{~mm} \mathrm{Hg} \mathrm{ml}^{-1}\right)$} \\
\hline & & P-value & $\beta$ coefficient & P-value & & P-value & $\beta$ coefficient & P-value \\
\hline Age & -0.12 & 0.47 & 0.20 & 0.20 & 0.26 & 0.11 & 0.46 & 0.002 \\
\hline Body mass index & 0.34 & 0.03 & -0.56 & $<0.001$ & -0.39 & 0.014 & -0.17 & 0.29 \\
\hline Creatinine & 0.21 & 0.2 & -0.30 & 0.054 & -0.29 & 0.08 & -0.08 & 0.64 \\
\hline Systolic BP & -0.06 & 0.72 & 0.38 & 0.013 & 0.43 & 0.006 & 0.32 & 0.042 \\
\hline Total cholesterol & -0.40 & 0.013 & 0.23 & 0.15 & 0.29 & 0.08 & 0.003 & 0.99 \\
\hline MHT vs. HHT status & -0.06 & 0.72 & 0.11 & 0.50 & 0.07 & 0.67 & -0.17 & 0.30 \\
\hline
\end{tabular}

Abbreviations: BP, blood pressure; Ea, arterial elastance; Eal, arterial elastance index; Ea/Ees, arterial-ventricular interaction; EeD, end-diastolic elastance; Ees, end-systolic elastance; HHT, 'highrisk' hypertension; MHT, malignant hypertension.

indicate that rigorous BP control may preserve or restore normal arterial-ventricular interactions.

\section{Limitations}

The study groups are fairly small in size because of scarcity of patients with MHT and strict inclusion/exclusion criteria employed in the study. Nevertheless the analyzed groups are well matched for most demographic and clinical parameters and meet the minimal requirements of the power calculation.

\section{Conclusion}

The study results indicate that despite previously reported abnormalities in endothelial functionality and pulse wave velocity patients with MHT and fairly well-controlled BP have values of the cardiac and Ea comparable to those with 'high-risk' hypertension. These results suggest that adequate BP control in MHT patients allows preservation or restoration of normal arterial-ventricular coupling.

\section{ACKNOWLEDGEMENTS}

This study was supported by $£ 20000$ Grant (Phillip Poole-Wilson seed corn fund) from the BUPA foundation (UK).

1 Pries AR, Reglin B, Secomb TW. Remodeling of blood vessels: responses of diameter and wall thickness to hemodynamic and metabolic stimuli. Hypertension 2005; 46: 725-731.

2 Aurigemma GP, Silver KH, Priest MA, Gaasch WH. Geometric changes allow normal ejection fraction despite depressed myocardial shortening in hypertensive left ventricular hypertrophy. J Am Coll Cardiol 1995; 26: 195-202.

3 Rosen BD, Edvardsen T, Lai S, Castillo E, Pan L, Jerosch-Herold M, Sinha S, Kronmal R, Arnett D, Crouse III JR, Heckbert SR, Bluemke DA, Lima JA. Left ventricular concentric remodeling is associated with decreased global and regional systolic function: the Multi-Ethnic Study of Atherosclerosis. Circulation 2005; 112 : 984-991.

4 Neumann T, Vollmer A, Schaffner T, Hess OM, Heusch G. Diastolic dysfunction and collagen structure in canine pacing-induced heart failure. J Mol Cell Cardiol 1999; 31: 179-192.

5 Hundley WG, Kitzman DW, Morgan TM, Hamilton CA, Darty SN, Stewart KP, Herrington DM, Link KM, Little WC. Cardiac cycle-dependent changes in aortic area and distensibility are reduced in older patients with isolated diastolic heart failure and correlate with exercise intolerance. J Am Coll Cardiol 2001; 38: 796-802.

6 Ishihara H, Yokota M, Sobue T, Saito H. Relation between ventriculoarterial coupling and myocardial energetics in patients with idiopathic dilated cardiomyopathy. J Am Coll Cardiol 1994; 23: 406-416.

7 Lane DA, Lip GY, Beevers DG. Improving survival of malignant hypertension patients over 40 years. Am J Hypertens 2009; 22: 1199-1204.

8 Shantsila A, Dwivedi G, Shantsila E, Butt M, Beevers DG, Lip GY. Persistent macrovascular and microvascular dysfunction in patients with malignant hypertension. Hypertension 2011; 57: 490-496.

9 Ventura HO, Mehra MR, Messerli FH. Desperate diseases, desperate measures: tackling malignant hypertension in the 1950s. Am Heart J 2001; 142: 197-203.

10 Dahlöf B, Sever PS, Poulter NR, Wedel H, Beevers DG, Caulfield M, Collins R, Kjeldsen SE, Kristinsson A, McInnes GT, Mehlsen J, Nieminen M, O'Brien E, Ostergren $J$. Prevention of cardiovascular events with an antihypertensive regimen of amlodipine adding perindopril as required versus atenolol adding bendroflumethiazide as required, in the Anglo-Scandinavian Cardiac Outcomes Trial-Blood Pressure Lowering Arm (ASCOT-BPLA): a multicentre randomised controlled trial. Lancet 2005; 366: 895-906.

11 Chen CH, Fetics B, Nevo E, Rochitte CE, Chiou KR, Ding PA, Kawaguchi M, Kass DA. Noninvasive single-beat determination of left ventricular end-systolic elastance in humans. J Am Coll Cardiol 2001; 38: 2028-2034.

12 Ommen SR, Nishimura RA, Appleton CP, Miller FA, Oh JK, Redfield MM, Tajik AJ. Clinical utility of Doppler echocardiography and tissue Doppler imaging in the estimation of left ventricular filling pressures: a comparative simultaneous Doppler-catheterization study. Circulation 2000; 102: 1788-1794.

13 Chen CH, Nakayama M, Nevo E, Fetics BJ, Maughan WL, Kass DA. Coupled systolicventricular and vascular stiffening with age: implications for pressure regulation and cardiac reserve in the elderly. J Am Coll Cardiol 1998; 32: 1221-1227.

14 Olivetti G, Melissari M, Balbi T, Quaini F, Cigola E, Sonnenblick EH, Anversa P. Myocyte cellular hypertrophy is responsible for ventricular remodelling in the hypertrophied heart of middle aged individuals in the absence of cardiac failure. Cardiovasc Res 1994; 28: 1199-1208.

15 Kelly RP, Ting CT, Yang TM, Liu CP, Maughan WL, Chang MS, Kass DA. Effective arterial elastance as index of arterial vascular load in humans. Circulation 1992; 86: 513-521.

16 Chantler PD, Lakatta EG, Najjar SS. Arterial-ventricular coupling: mechanistic insights into cardiovascular performance at rest and during exercise. J Appl Physiol 2008; 105: 1342-1351.

17 Leite-Moreira AF, Correia-Pinto J, Gillebert TC. Afterload induced changes in myocardial relaxation: a mechanism for diastolic dysfunction. Cardiovasc Res 1999; 43 : 344-353.

18 Borlaug BA, Kass DA. Ventricular-vascular interaction in heart failure. Heart Fail Clin 2008; 4: 23-36.

19 Kass DA, Maughan WL, Guo ZM, Kono A, Sunagawa K, Sagawa K. Comparative influence of load versus inotropic states on indexes of ventricular contractility: experimental and theoretical analysis based on pressure-volume relationships. Circulation 1987; 76: 1422-1436. 